\title{
Generalized Kac lemma for recurrence time in iterated open quantum systems
}

\author{
P. Sinkovicz, T. Kiss, and J. K. Asbóth \\ Institute for Solid State Physics and Optics, Wigner Research Centre, Hungarian Academy of Sciences, \\ P.O. Box 49, H-1525 Budapest, Hungary \\ (Received 10 November 2015; revised manuscript received 23 March 2016; published 9 May 2016)
}

\begin{abstract}
We consider recurrence to the initial state after repeated actions of a quantum channel. After each iteration a projective measurement is applied to check recurrence. The corresponding return time is known to be an integer for the special case of unital channels, including unitary channels. We prove that for a more general class of quantum channels the expected return time can be given as the inverse of the weight of the initial state in the steady state. This statement is a generalization of the Kac lemma for classical Markov chains.
\end{abstract}

DOI: 10.1103/PhysRevA.93.050101

\section{INTRODUCTION}

According to the Poincaré recurrence theorem [1,2], any closed classical physical system, when observed for a sufficiently long time, will return arbitrarily close to its initial state. The extension of this statement to open systems whose dynamics can be modeled as Markov chains $[3,4]$ is given by the Kac lemma [5]. This connects the expected return time to the limit distribution, i.e., the probability distribution of the system in the infinite-time limit. The expected return time (Poincare time) is the inverse of the weight of the initial state in the limit distribution. If the weight vanishes, the expected return time diverges, and the probability of return is less than 1.

A version of the Poincaré recurrence theorem also holds for closed quantum-mechanical systems, which are periodically measured to test whether recurrence has occurred. Here, the system is assumed to start from an initial state given by a wave function, which is evolved by the same unitary time-step operator between successive measurements. Every measurement influences the dynamics: It either signals a return, and projects the system to the initial state, or signals no return, and projects out the initial state from the wave function. Grünbaum et al. [6] have shown that in this case the expected return time (number of measurements) is either infinite, or it is an integer. We generalized [7] this result to open quantum-mechanical systems whose dynamics is given by a quantum channel [8-12]. We found that if the channel is unital, i.e., if the limit distribution is the completely mixed state in an effective Hilbert space that contains the initial state [13-15], then the expected return time is equal to the dimensionality of that effective Hilbert space.

One cannot help but notice the fact that the expected return time in open quantum systems with unital dynamics is an integer in accordance with the Kac lemma. Indeed, the dimensionality of the effective Hilbert space is the inverse of the weight of the initial state in the (appropriately defined) limit distribution of the dynamics. The question arises: Can we push this statement further, and ask if there is a broader class of open quantum dynamical systems for which a quantum Kac lemma would hold? In this Rapid Communication we answer this question positively. We show that for a rather general type of quantum channel the expected return time can be calculated from the related steady state of the system.
The structure of this Rapid Communication is as follows. Section II contains the definitions and ideas that we need to set up the problem. We state our result in Sec. III. In Sec. IV we give a possible application of our theorem. Section V concludes with a discussion of the results and some potentially interesting questions.

\section{DEFINITIONS}

We consider discrete-time dynamics of an open quantum system. The state of the system is described by a density operator $\hat{\varrho}(t)$, representing a mixture of pure states from an $N$ dimensional Hilbert space $\mathcal{H}$, with $t \in \mathbb{N}$ denoting the discrete time. Starting from a pure initial state $|\Psi\rangle \in \mathcal{H}$, we obtain the state by iterations of the fixed time-step superoperator $\mathcal{S}[\cdot]$,

$$
\hat{\varrho}(t)=\mathcal{S}^{t}[|\Psi\rangle\langle\Psi|] .
$$

As usual, we assume $\mathcal{S}[\cdot]$ to be a linear, trace-preserving, and completely positive map, i.e., a quantum channel. It can thus be written using Kraus operators [16-20] as

$$
\mathcal{S}[\hat{\varrho}]=\sum_{\nu=0}^{r} \hat{A}_{\nu} \hat{\varrho} \hat{A}_{\nu}^{\dagger},
$$

where $r \leqslant N^{2}$ is the Kraus rank of $\mathcal{S}[\cdot]$, and $\hat{A}_{v}: \mathcal{H} \rightarrow \mathcal{H}$ are the Kraus operators, with the normalization

$$
\sum_{v} \hat{A}_{v}^{\dagger} \hat{A}_{v}=\hat{\mathbb{I}},
$$

where $\hat{I}$ represents the unit operator on $\mathcal{H}$.

We can construct a steady state of the dynamics from the initial state $|\Psi\rangle$ as

$$
\hat{\chi}=\lim _{T \rightarrow \infty} \frac{1}{T} \sum_{t=0}^{T-1} \mathcal{S}^{t}[|\Psi\rangle\langle\Psi|] .
$$

This limit is always well defined since we are in a finitedimensional Hilbert space [20]. The operator $\hat{\chi}$ is a convex mixture of density operators, and is thus self-adjoint, positive, and fulfills $\operatorname{Tr}[\hat{\chi}]=1$ : It can be interpreted as a density operator of the system. It represents a steady state, since

$$
\mathcal{S}[\hat{\chi}]=\hat{\chi}-\lim _{T \rightarrow \infty} \frac{1}{T}|\Psi\rangle\langle\Psi|=\hat{\chi} .
$$




\section{First return time}

To measure the first return time, we need to disturb the dynamics $[21,22]$. We follow each time step by a dichotomic measurement that checks whether the system has returned to the state $|\Psi\rangle\langle\Psi|$, or if it is in the orthogonal subspace $\mathcal{H}_{\perp}$, defined by

$$
|\Phi\rangle \in \mathcal{H}_{\perp} \Leftrightarrow\langle\Phi \mid \Psi\rangle=0 .
$$

The postmeasurement state corresponding to "no return" is obtained using the projector

$$
\mathcal{M}[\hat{\varrho}]=(\hat{\mathbb{I}}-|\Psi\rangle\langle\Psi|) \hat{\varrho}(\hat{\mathbb{I}}-|\Psi\rangle\langle\Psi|) .
$$

Note that the projector $\mathcal{M}[\cdot]$ does not conserve the trace: Its outcome is a conditional density operator, whose trace represents the probability that the particle described by $\hat{\varrho}$ has not returned. The modified dynamics, including the dichotomic measurements, is defined by

$$
\hat{\varrho}_{\text {cond }}(t)=(\mathcal{M S})^{t}[|\Psi\rangle\langle\Psi|] \text {. }
$$

Here, the trace of the conditional density operator is a probability,

$$
\operatorname{Tr} \hat{\varrho}_{\text {cond }}(t)=\mathrm{P}(\text { no return up until time } t) .
$$

We call the iterated open quantum channel recurrent when started from $|\Psi\rangle$, if it returns with probability 1 in the sense defined above, i.e., if $\lim \operatorname{Tr} \hat{\varrho}_{\text {cond }}(t)=0$. This is in line with Ref. [6]. In the rest of this Rapid Communication we will only concern ourselves with recurrent channels.

Let us remark that the states of a system evolving from the initial state $|\Psi\rangle$, either by the evolution operator $\mathcal{S}[\cdot]$ or by $\mathcal{M S}[\cdot]$, may explore only a subspace of $\mathcal{H}$, but they span the same subspace, i.e., the relevant Hilbert space $\mathcal{H}_{\text {rel }}$, as we have shown previously [7].

The expected return time $\overline{\mathrm{T}}_{\Psi}$ is the expectation value of the first return time, calculated using the probabilities in Eq. (9). Whenever the iterated open quantum channel is recurrent when started from $|\Psi\rangle$, the expected return time can be expressed using the sum of the conditional density operators [7], which we denote by

$$
\hat{\varrho}_{\text {cond }}=\lim _{T \rightarrow \infty} \sum_{t=0}^{T} \hat{\varrho}_{\text {cond }}(t) .
$$

Note that, unlike in Eq. (4) for the steady state, there is no factor of $1 / T$ here, and so this sum can diverge. If the sum is divergent, then the expected return time is also divergent, otherwise the trace of $\hat{\varrho}_{\text {cond }}$ gives the expected return time,

$$
\overline{\mathrm{T}}_{\Psi}=\operatorname{Tr} \hat{\varrho}_{\text {cond }} .
$$

In Ref. [7] we have shown that in the case of unital dynamics, where all eigenvalues of $\hat{\chi}$ are equal, the expected return time $\bar{T}_{\Psi}$ can be obtained from the steady state $\hat{\chi}$. In that case, we found that the expected return time is an integer, equal to the dimensionality of $\hat{\chi}$. In the next section we generalize this result, and give a formula that connects the expected return time $\overline{\mathrm{T}}_{\Psi}$ to the steady state $\hat{\chi}$ for a broader class of dynamical systems.

\section{QUANTUM KAC LEMMA}

We now formulate the main result of this Rapid Communication. If the initial state $|\Psi\rangle$ is an eigenvector of the steady state $\hat{\chi}$, with nonzero eigenvalue $\lambda \in \mathbb{R}$, then the expected return time is $\bar{T}_{\Psi}=1 / \lambda$. In formulas,

$$
\hat{\chi}|\Psi\rangle=\lambda|\Psi\rangle, \quad \lambda \neq 0 \Longrightarrow \overline{\mathrm{T}}_{\Psi}=\frac{1}{\langle\Psi|\hat{\chi}| \Psi\rangle} .
$$

This is a direct generalization of the classical Kac lemma [5], where the expected first return time to site $n$ is the reciprocal of the corresponding component $\pi_{n}$ of the equilibrium distribution vector $\pi$. We therefore refer to Eq. (12) as the quantum Kac lemma.

Note that $\lambda \neq 0$ ensures that $\lim \hat{\varrho}_{\text {cond }}(t)=0$, proved in the Appendix.

\section{Proof}

The quantum Kac lemma is a direct consequence of the statement

$$
\hat{\chi}|\Psi\rangle=\lambda|\Psi\rangle \Leftrightarrow \hat{\varrho}_{\text {cond }}=\frac{1}{\langle\Psi|\hat{\chi}| \Psi\rangle} \hat{\chi} .
$$

This states that the sum $\hat{\varrho}_{\text {cond }}$ of the conditional density operators, Eq. (10), is proportional to the steady state $\hat{\chi}$ of Eq. (4), if and only if $|\Psi\rangle$ is an eigenstate of $\hat{\chi}$. The trace of the relation on the right-hand side of Eq. (13), using Eq. (11), gives directly the quantum Kac lemma, Eq. (12). It remains to show that Eq. (13) holds.

We now prove Eq. (13) in two steps.

First, we show that whenever $\hat{\varrho}_{\text {cond }}$ is proportional to $\hat{\chi}$, then the initial state is one of the eigenvectors of $\hat{\chi}$. This statement follows from the fact that we can express $\hat{\varrho}_{\text {cond }}$ as

$$
\hat{\varrho}_{\text {cond }}=|\Psi\rangle\langle\Psi|+\lim _{T \rightarrow \infty} \sum_{t=1}^{T}(\mathcal{M S})^{t}[|\Psi\rangle\langle\Psi|] .
$$

The conditional dynamics maps any density operator $\hat{\varrho}$ to the orthogonal subspace, that is, $\mathcal{M S}[\hat{\varrho}]: \mathcal{H} \rightarrow \mathcal{H}_{\perp}$. Furthermore, $\hat{\varrho}_{\text {cond }}$ is proportional to the steady state, hence by applying Eq. (14) to the initial state $|\Psi\rangle$, we can establish the following,

$$
\lambda=\langle\Psi|\hat{\chi}| \Psi\rangle .
$$

This concludes the first step of the proof.

The second step in the proof of Eq. (13) is to show that, whenever the initial state $|\Psi\rangle$ is an eigenstate of the density operator of the steady state $\hat{\chi}$, then $\hat{\varrho}_{\text {cond }}$ is proportional to the steady state. We notice that if $|\Psi\rangle$ is an eigenvector of $\hat{\chi}=\hat{\chi}^{\dagger}$, with eigenvalue $\lambda \neq 0$, then the steady state is given by

$$
\hat{\chi}=\lambda|\Psi\rangle\langle\Psi|+\hat{\chi}_{\perp},
$$

where $\hat{\chi}_{\perp}=\mathcal{M}[\hat{\chi}]$. On the other hand, $\hat{\chi}$ is a steady state of the dynamics [see Eq. (5)], i.e., $\hat{\chi}=\mathcal{S}[\hat{\chi}]$. Projecting both sides of this latter equation using $\mathcal{M}[\cdot]$, inserting Eq. (16), and rearranging gives us

$$
\mathcal{M S}[|\Psi\rangle\langle\Psi|]=\frac{1}{\lambda}\left(\hat{\chi}_{\perp}-\mathcal{M S}\left[\hat{\chi}_{\perp}\right]\right) .
$$


Finally, inserting Eq. (17) in Eq. (14) gives us

$$
\begin{aligned}
\hat{\varrho}_{\text {cond }} & =|\Psi\rangle\langle\Psi|+\frac{1}{\lambda} \lim _{T \rightarrow \infty} \sum_{t=0}^{T}(\mathcal{M S})^{t}\left[\hat{\chi}_{\perp}-\mathcal{M S}\left[\hat{\chi}_{\perp}\right]\right] \\
& =\frac{1}{\lambda} \hat{\chi}-\frac{1}{\lambda} \lim _{T \rightarrow \infty}(\mathcal{M S})^{T}\left[\hat{\chi}_{\perp}\right] .
\end{aligned}
$$

To finish the proof of the second step, we need to show that

$$
\lim _{t \rightarrow \infty}(\mathcal{M S})^{t}\left[\hat{\chi}_{\perp}\right]=0 .
$$

This will be enough because, by assumption, $\lambda \neq 0$. It is clear that $\hat{\chi}_{\perp}$ is an unnormalized density operator in the relevant Hilbert space. In fact, using the results of Ref. [23], it can be shown that the relevant Hilbert space is already spanned by the first $N$ states in the orbit, i.e., by the states $\hat{\varrho}_{\text {cond }}(n)$, with $n=0, \ldots, N-1$, where $N$ is the dimensionality of the relevant Hilbert space. In formulas,

$$
\hat{\chi}_{\perp}=\sum_{n=0}^{N-1} c_{n}(\mathcal{M S})^{n}[|\Psi\rangle\langle\Psi|]
$$

with some complex coefficients $c_{n} \in \mathbb{C}$. Now consider applying $(\mathcal{M S})^{t}[\cdot]$ to this equation, and take the limit $t \rightarrow \infty$. As we show in the Appendix, each term on the right-hand side vanishes. Therefore, the finite sum also vanishes, and so we have Eq. (19). This completes the proof of Eq. (13), and thus of the quantum Kac lemma.

\section{EXAMPLE: EVALUATING HITTING TIME VIA CLASSICAL MONITORING}

Besides the class of unital dynamics, the quantum Kac lemma also applies to any system where during each time step the initial state $|\Psi\rangle$ is interfaced to the rest of the system only by incoherent processes. More specifically, it applies when the time-step operator $\mathcal{S}[\cdot]$ can be split into three parts,

$$
\hat{\varrho}(t+1)=\mathcal{S}[\hat{\varrho}(t)]=\mathcal{D}_{\text {out }}\left[\mathcal{T}_{\perp}\left[\mathcal{D}_{\text {in }}[\hat{\varrho}(t)]\right]\right] .
$$

Here, $\mathcal{T}_{\perp}[\cdot]$ is a superoperator that acts nontrivially only in $\mathcal{H}_{\perp}$, defined in Eq. (6), i.e.,

$$
\mathcal{T}_{\perp}[\hat{\varrho}]=|\Psi\rangle\langle\Psi|\hat{\varrho}| \Psi\rangle\langle\Psi|+\sum_{v} \hat{K}_{v} \hat{\varrho} \hat{K}_{v}^{\dagger},
$$

with Kraus operators $\hat{K}_{v}: \mathcal{H} \rightarrow \mathcal{H}_{\perp}$. The superoperators $\mathcal{D}_{\text {in }}[\cdot]$ and $\mathcal{D}_{\text {out }}[\cdot]$ describe the incoherent particle transfer from $|\Psi\rangle$ to the rest of the system and vice versa,

$$
\begin{aligned}
& \mathcal{D}_{\text {in }}[\hat{\varrho}]=\sum_{\nu=1}^{N} p_{\nu}\left|\Phi_{\nu}\right\rangle\langle\Psi|\hat{\varrho}| \Psi\rangle\left\langle\Phi_{\nu}\right|+\hat{A}_{\text {in }} \hat{\varrho} \hat{A}_{\text {in }}, \\
& \hat{A}_{\text {in }}=\sqrt{\hat{\mathbb{I}}-\sum_{\nu=1}^{N} p_{\nu}|\Psi\rangle\langle\Psi|,} \\
& \mathcal{D}_{\text {out }}[\hat{\varrho}]=\sum_{\mu=1}^{M} q_{\mu}|\Psi\rangle\left\langle\alpha_{\mu}|\hat{\varrho}| \alpha_{\mu}\right\rangle\langle\Psi|+\hat{A}_{\text {out }} \hat{\varrho} \hat{A}_{\text {out }}, \\
& \hat{A}_{\text {out }}=\sqrt{\hat{\mathbb{I}}-\sum_{\mu=1}^{M} q_{\mu}\left|\alpha_{\mu}\right\rangle\left\langle\alpha_{\mu}\right|} .
\end{aligned}
$$

Here, the rates $p_{\nu}, q_{\mu}$ are assumed to be positive, and $\sum p_{\nu} \leqslant$ 1 , and $\sum q_{\mu} \leqslant 1$, and the states $\left|\Phi_{\nu}\right\rangle,\left|\alpha_{\mu}\right\rangle \in \mathcal{H}_{\perp}$. In this case, the condition in Eq. (12) is automatically satisfied, therefore we can apply the quantum Kac lemma and determine $\overline{\mathrm{T}}_{\Psi}$ as the inverse of the weight of the initial state $|\Psi\rangle$ in the steady state $\hat{\chi}$.

The example above can be used to express the hitting time for an iterated quantum dynamical system in terms of a stationary distribution. For this, we let $\mathcal{H}_{\perp}$ denote the Hilbert space where this quantum dynamics take place, and $\left|\Phi_{1}\right\rangle$ and $\left|\alpha_{1}\right\rangle$ denote the pure states from which and into which the hitting time is sought. We extend the Hilbert space by the extra ancilla state $|\Psi\rangle$, set $N=1, p_{1}=1$, and $M=1, q_{1}=1$. The hitting time from $\left|\Phi_{1}\right\rangle$ to $\left|\alpha_{1}\right\rangle$ is then given by the expected return time to $|\Psi\rangle$.

\section{DISCUSSION}

We found a relationship between the return time to an initial pure state and the steady state for an iterated quantum channel. If the initial state is an eigenvector of that steady state, then the reciprocal of the corresponding eigenvalue gives us the expected return time for that particular initial state. This is not only a generalization of the results of Ref. [7] for unital channels (which includes unitary dynamics), but also of the Kac lemma about Markov chains.

The condition $\hat{\chi}|\Psi\rangle=\lambda|\Psi\rangle$ is sufficient, but not necessary, for the form of the expected return time on the right-hand side of Eq. (12) to hold. An example is given by the so-called classical-quantum channels [24], defined by the evolution equation

$$
\hat{\varrho}_{\mathrm{cq}}(t+1)=\mathcal{S}_{\mathrm{cq}}[\hat{\varrho}(t)]=\sum_{n=1}^{\operatorname{dim} \mathcal{H}}\left\langle\varphi_{n}|\hat{\varrho}(t)| \varphi_{n}\right\rangle \hat{\sigma}_{n},
$$

where the generators of the dynamics $\left\{\hat{\sigma}_{n}\right\}$ are positive and self-adjoint operators with unit trace, and the Hilbert-space vectors $\left\{\left|\varphi_{n}\right\rangle\right\}$ are an orthonormal set (we note that a similar, but not equivalent, notion of a classical-quantum channel was introduced in Ref. [25]). The speciality of this type of dynamics can be understood if we restrict our attention only to the dynamics of the diagonal elements. The matrix elements within the diagonal are transformed among themselves by a timeindependent transition matrix $\hat{W}$, where $W_{m, n}=\left\langle\varphi_{m}\left|\hat{\sigma}_{n}\right| \varphi_{m}\right\rangle$ gives the probability that the system makes the $\left|\varphi_{n}\right\rangle \rightarrow\left|\varphi_{m}\right\rangle$ transition. Based on $\hat{W}$, one can naturally construct a classical homogeneous Markov chain for which the original Kac lemma is valid and has the same recurrence properties. One can generalize the previous example, and say that Eq. (12) holds for every random walk, where the evolution of the diagonal elements depends only on other diagonal elements. In these cases the dynamics of the diagonal elements can be separated from the dynamics of the off-diagonal elements, and their evolution can be described as a discrete-time classical random walk for which the classical Kac lemma can be applied.

In our generalization of the Kac lemma as well as in the example of the classical-quantum channel, the steady state corresponding to the given initial state fully determines the expected return time. It would be fascinating to know whether there are some even more general classes of quantum channels 
for which the knowledge of the steady state is enough to calculate the first return time.

Let us note that there are alternative ways to define a return time. One can avoid the disturbance of the measurement, for example, by taking a new system from an ensemble after each measurement. The Pólya number for quantum walks characterizing recurrence has been defined in this way [26,27]. There are also alternative ways to define iterative open quantum dynamics, e.g., the "open quantum random walks" [28], for which there are known results on recurrence and return time [25].

Our theorem proved to be a useful tool to determine the hitting time for an arbitrary iterated quantum channel by applying an extra monitoring site coupled classically to the initial and final states of the system to be observed. Monitoring the hitting time in this way is a discrete-time analog for the hitting time defined for continuous-time quantum walks, which is defined through the survival time of an excitation in the system where the Hamiltonian includes a trapping site [29-31].

\section{ACKNOWLEDGMENTS}

We thank Gernot Alber, András Frigyik, and Melinda Herbáth for stimulating discussions. We acknowledge support by the Hungarian Scientific Research Fund (OTKA) under Contracts No. K83858 and No. NN109651, the Hungarian Academy of Sciences (Lendület Program, LP2011-016), and the Deutscher Akademischer Austauschdienst (TempusDAAD Project No. 65049). J.K.A. was supported by the Janos Bolyai Scholarship of the Hungarian Academy of Sciences.

\section{APPENDIX: RECURRENCE OF THE INITIAL STATE}

In this Appendix we prove that if the initial state $|\Psi\rangle$ is one of the eigenvectors of the steady state $\hat{\chi}$ of Eq. (4) with eigenvalue $\lambda \neq 0$, then $\lim \hat{\varrho}_{\text {cond }}(t)=0$, or in other words, the process is recurrent (returns with probability 1), i.e.,

$$
\hat{\chi}|\Psi\rangle=\lambda|\Psi\rangle, \quad \lambda \neq 0 \Rightarrow \lim _{t \rightarrow \infty}(\mathcal{M S})^{t}[|\Psi\rangle\langle\Psi|]=0 .
$$

For the proof, we use a steady state of the operator $\mathcal{M S}[\cdot]$ defined as

$$
\hat{\chi}_{\mathcal{M}}=\lim _{T \rightarrow \infty} \frac{1}{T} \sum_{t=0}^{T-1}(\mathcal{M S})^{t}[|\Psi\rangle\langle\Psi|] .
$$

The operator $\hat{\chi}_{\mathcal{M}}$ is a non-negative, Hermitian operator, and it is nonvanishing if $\lim \operatorname{Tr}\left\{(\mathcal{M S})^{t}[|\Psi\rangle\langle\Psi|]\right\}>C \in \mathbb{R}^{+}$. The operator $\hat{\chi}_{\mathcal{M}}$ is obviously an unnormalized steady state of $\mathcal{M S}[\cdot]$, i.e.,

$$
\mathcal{M S}\left[\hat{\chi}_{\mathcal{M}}\right]=\hat{\chi}_{\mathcal{M}}
$$

Taking the expectation value of the two sides of this relation in the state $|\Psi\rangle$ tells us

$$
\left\langle\Psi\left|\hat{\chi}_{\mathcal{M}}\right| \Psi\right\rangle=0 .
$$

On the other hand, $\left\langle\Psi\left|\mathcal{S}\left[\hat{\chi}_{\mathcal{M}}\right]\right| \Psi\right\rangle=0$ must hold as well, or else the trace of $\hat{\chi}_{\mathcal{M}}$ would decrease under the mapping $\mathcal{M S}[\cdot]$. Therefore, $\hat{\chi}_{\mathcal{M}}$ is not only a steady state of $\mathcal{M S}[\cdot]$, but also of $\mathcal{S}[\cdot]$, i.e.,

$$
\mathcal{S}\left[\hat{\chi}_{\mathcal{M}}\right]=\hat{\chi}_{\mathcal{M}}
$$

We remark that the operator $\mathcal{M}[\cdot]$ does not take us out of the relevant Hilbert space [7], and thus the operator $\hat{\chi}_{\mathcal{M}}$ has all its support in the relevant Hilbert space.

We will prove Eq. (A1) below, by showing that $\operatorname{Tr} \hat{\chi}_{\mathcal{M}}=0$.

\section{Theoretical tools: Decaying subspace, minimal enclosures}

In the proof, we will use the concepts of minimal enclosures, and of the decaying subspace, as introduced in Ref. [32]. We recapitulate the definitions, and the basic properties, below.

The decaying subspace $\mathcal{D}$ is defined as

$$
\mathcal{D}=\left\{|\varphi\rangle \in \mathcal{H}: \forall \hat{\varrho} \lim _{t \rightarrow \infty}\left\langle\varphi\left|\mathcal{S}^{t}[\hat{\varrho}]\right| \varphi\right\rangle=0\right\},
$$

i.e., it is spanned by the states which have a vanishing overlap in the long-time limit with any density operator $\hat{\varrho}$ which acts in $\mathcal{H}$. We will use $\mathcal{R}$ to denote its complement, i.e.,

$$
\mathcal{R}=\left\{|\varphi\rangle \in \mathcal{H}: \forall\left|\varphi_{\mathcal{D}}\right\rangle \in \mathcal{D}:\left\langle\varphi \mid \varphi_{\mathcal{D}}\right\rangle=0\right\} .
$$

Time evolution by $\mathcal{S}[\cdot]$ does not lead out of the set $\mathcal{R}$, i.e., $\forall t \in \mathbb{N},\left|\varphi_{\mathcal{R}}\right\rangle \in \mathcal{R},\left|\varphi_{\mathcal{D}}\right\rangle \in \mathcal{D}:$

$$
\left\langle\varphi_{\mathcal{D}}\left|\mathcal{S}^{t}\left[\left|\varphi_{\mathcal{R}}\right\rangle\left\langle\varphi_{\mathcal{R}}\right|\right]\right| \varphi_{\mathcal{D}}\right\rangle=0 .
$$

This property of $\mathcal{R}$ defines it to be an enclosure [32]. As every enclosure, the space $\mathcal{R}$ can be written as the sum of orthogonal minimal enclosures [32],

$$
\mathcal{R}=\mathcal{R}_{1} \oplus \mathcal{R}_{2} \oplus \cdots \oplus \mathcal{R}_{M}
$$

\section{Proof}

We begin by showing that the initial state $|\Psi\rangle$ lies in the subspace $\mathcal{R}$, i.e.,

$$
|\Psi\rangle \in \mathcal{R}
$$

We show this by splitting the initial state into components from the two subspaces,

$$
|\Psi\rangle=c_{\mathcal{D}}\left|\Psi_{\mathcal{D}}\right\rangle+c_{\mathcal{R}}\left|\Psi_{\mathcal{R}}\right\rangle,
$$

where $c_{\mathcal{D}}, c_{\mathcal{R}} \in \mathbb{C},\left|\Psi_{\mathcal{D}}\right\rangle \in \mathcal{D}$, and $\left|\Psi_{\mathcal{R}}\right\rangle \in \mathcal{R}$. Using this decomposition, we have

$$
\left\langle\Psi_{\mathcal{D}}|\hat{\chi}| \Psi\right\rangle=c_{\mathcal{D}}\left\langle\Psi_{\mathcal{D}}|\hat{\chi}| \Psi_{\mathcal{D}}\right\rangle+c_{\mathcal{R}}\left\langle\Psi_{\mathcal{D}}|\hat{\chi}| \Psi_{\mathcal{R}}\right\rangle
$$

where $\hat{\chi}$ is the steady state of $\mathcal{S}[\cdot]$ defined by Eq. (4). The first term on the right-hand side vanishes, since $\hat{\chi}$ is a fixed point of $\mathcal{S}[\cdot]$, and so it can have no weight in the decaying subspace, We can bound the second term from above using the Schwarz inequality for the vectors $\hat{\chi}^{1 / 2}\left|\Psi_{\mathcal{D}}\right\rangle$ and $\hat{\chi}^{1 / 2}\left|\Psi_{\mathcal{R}}\right\rangle$, whereby

$$
\left|\left\langle\Psi_{\mathcal{D}}|\hat{\chi}| \Psi_{\mathcal{R}}\right\rangle\right|^{2} \leqslant\left\langle\Psi_{\mathcal{D}}|\hat{\chi}| \Psi_{\mathcal{D}}\right\rangle\left\langle\Psi_{\mathcal{R}}|\hat{\chi}| \Psi_{\mathcal{R}}\right\rangle=0,
$$

where we used the positivity of $\hat{\chi}$ as well as the fact that $\left\langle\Psi_{\mathcal{D}}|\hat{\chi}| \Psi_{\mathcal{D}}\right\rangle=0$. Thus, $\left\langle\Psi_{\mathcal{D}}|\hat{\chi}| \Psi\right\rangle=0$. On the other hand, since $\hat{\chi}|\Psi\rangle=\lambda|\Psi\rangle$, we have

$$
\left\langle\Psi_{\mathcal{D}}|\hat{\chi}| \Psi\right\rangle=\lambda c_{\mathcal{D}}\left\langle\Psi_{\mathcal{D}} \mid \Psi_{\mathcal{D}}\right\rangle+\lambda c_{\mathcal{R}}\left\langle\Psi_{\mathcal{D}} \mid \Psi_{\mathcal{R}}\right\rangle=\lambda c_{\mathcal{D}},
$$

where we used the orthogonality of $\mathcal{D}$ and $\mathcal{R}$, as well as the normalization of the vectors $\left|\Psi_{\mathcal{D}}\right\rangle$ and $\left|\Psi_{\mathcal{R}}\right\rangle$. Comparing this last result with $\left\langle\Psi_{\mathcal{D}}|\hat{\chi}| \Psi\right\rangle=0$ gives us $c_{\mathcal{D}}=0$, or, equivalently, $|\Psi\rangle \in \mathcal{R}$, which is Eq. (A10), the first step.

Since $|\Psi\rangle \in \mathcal{R}$, the relevant Hilbert space is the sum of a subset of the minimal enclosures, those that are not orthogonal 
to the initial state $|\Psi\rangle$. We let these be the first $M^{\prime}$ minimal orthogonal enclosures. We can then split the initial states into components from these enclosures,

$$
|\Psi\rangle=c_{1}\left|\Psi_{1}\right\rangle+c_{2}\left|\Psi_{2}\right\rangle+\cdots+c_{M^{\prime}}\left|\Psi_{M^{\prime}}\right\rangle
$$

with $c_{j} \in \mathbb{C}$, and $c_{j} \neq 0$ for all $j=1, \ldots, M^{\prime}$. The unique steady states of $\mathcal{S}[\cdot]$ in each of these minimal enclosures is $\hat{\chi}_{j}$. Note that $\left\langle\Psi\left|\hat{\chi}_{j}\right| \Psi\right\rangle>0$ for all $j$. Any steady state of $\mathcal{S}[\cdot]$ in the relevant Hilbert space is a convex combination of the $\hat{\chi}_{j}$ [32]. Since $\hat{\chi}_{\mathcal{M}}$ is an unnormalized steady state of $\mathcal{S}[\cdot]$ in the relevant Hilbert space, it can be written as $\hat{\chi}_{\mathcal{M}}=\sum p_{j} \hat{\chi}_{j}$ with non-negative weights $p_{j} \geqslant 0$. Therefore,

$$
\left\langle\Psi\left|\hat{\chi}_{\mathcal{M}}\right| \Psi\right\rangle=\sum_{j=1}^{M^{\prime}} p_{j}\left\langle\Psi\left|\hat{\chi}_{j}\right| \Psi\right\rangle .
$$

Since $\left\langle\Psi\left|\hat{\chi}_{j}\right| \Psi\right\rangle>0$ for all $j$, we must have $p_{j}=0$ for all $j$, i.e., the operator $\hat{\chi}_{\mathcal{M}}$ of Eq. (A2) must vanish. This proves Eq. (A1).
[1] H. Poincaré, Acta Math. 13, A271 (1890).

[2] L. Barreira, in XIVth International Congress on Mathematical Physics (World Scientific, Singapore, 2006), pp. 415-422.

[3] D. Aldous and J. A. Fill, Reversible Markov Chains and Random Walks on Graphs (2002).

[4] J. G. Kemeny and J. L. Snell, Finite Markov Chains (van Nostrand, Princeton, NJ, 1960), Vol. 356.

[5] M. Kac, Bull. Am. Math. Soc. 53, 1002 (1947).

[6] F. A. Grünbaum, L. Velázquez, A. H. Werner, and R. F. Werner, Commun. Math. Phys. 320, 543 (2013).

[7] P. Sinkovicz, Z. Kurucz, T. Kiss, and J. K. Asbóth, Phys. Rev. A 91, 042108 (2015).

[8] H.-P. Breuer and F. Petruccione, The Theory of Open Quantum Systems (Oxford University Press, Oxford, UK, 2002).

[9] D. Chruściński, Open Syst. Inf. Dyn. 21, 1440004 (2014).

[10] R. Alicki and M. Fannes, Quantum Dynamical Systems (Oxford University Press, Oxford, UK, 2001).

[11] F. Benatti and R. Floreanini, Int. J. Mod. Phys. B 19, 3063 (2005).

[12] V. Paulsen, Completely Bounded Maps and Operator Algebras (Cambridge University Press, Cambridge, UK, 2002), Vol. 78.

[13] A. Arias, A. Gheondea, and S. Gudder, J. Math. Phys. (N.Y.) 43, 5872 (2002).

[14] J. A. Holbrook, D. W. Kribs, and R. Laflamme, Quantum Inf. Process. 2, 381 (2003).

[15] J. Novotný, G. Alber, and I. Jex, Cent. Eur. J. Phys. 8, 1001 (2010).

[16] K. Kraus, A. Böhm, and J. Dollard, States, Effects, and Operations Fundamental Notions of Quantum Theory, Lecture Notes in Physics Vol. 190 (Springer, Berlin, 1983).

[17] E. Sudarshan, P. Mathews, and J. Rau, Phys. Rev. 121, 920 (1961).
[18] M.-D. Choi, in Quantum Computation and Quantum Information Theory: Reprint Volume with Introductory Notes for ISI TMR Network School, 12-23 July 1999, Villa Gualino, Torino, Italy, edited by C. Machiavello, G. M. Palma, and A. Zeilinger (World Scientific, Singapore, 2000), p. 174.

[19] I. Bengtsson and K. Zyczkowski, Geometry of Quantum States: An Introduction to Quantum Entanglement (Cambridge University Press Cambridge, UK, 2006).

[20] M. M. Wolf, Quantum Channels \& Operations: Guided Tour, 2012.

[21] J. Kempe, Contemp. Phys. 44, 307 (2003).

[22] E. B. Davies, Quantum Theory of Open Systems (Academic Press, New York, 1976).

[23] J. Liesen and Z. Strakos, Krylov Subspace Methods: Principles and Analysis (Oxford University Press, Oxford, UK, 2012).

[24] M. E. Shirokov, Sb. Math. 204, 1215 (2013).

[25] C. F. Lardizabal and R. R. Souza, J. Stat. Phys. 159, 772 (2015).

[26] M. Štefaňák, I. Jex, and T. Kiss, Phys. Rev. Lett. 100, 020501 (2008).

[27] M. Štefaňák, T. Kiss, and I. Jex, Phys. Rev. A 78, 032306 (2008).

[28] S. Attal, F. Petruccione, and I. Sinayskiy, Phys. Lett. A 376, 1545 (2012).

[29] O. Mülken, A. Blumen, T. Amthor, C. Giese, M. ReetzLamour, and M. Weidemüller, Phys. Rev. Lett. 99, 090601 (2007).

[30] O. Mülken, V. Pernice, and A. Blumen, Phys. Rev. E 76, 051125 (2007).

[31] M. Varbanov, H. Krovi, and T. A. Brun, Phys. Rev. A 78, 022324 (2008).

[32] B. Baumgartner and H. Narnhofer, Rev. Math. Phys. 24, 1250001 (2012). 